\title{
Electron Distribution in Some Zincblende-Type Crystals*
}

\author{
Ryosei Uno, Haruo Ozawa, and Jun Ishigaki \\ College of Humanities and Sciences, Nihon University, 3-25-40, Sakurajosui Setagaya-ku, \\ Tokyo 156, Japan
}

\section{Ken Yukino}

National Institute for Research in Organic Materials, 1-1, Namiki, Tsukuba, Ibaragi 305, Japan

Z. Naturforsch. 48a, 38-40 (1993); received January 10, 1992

The elctron density distribution in crystalline $\mathrm{ZnSe}$ and $\mathrm{ZnTe}$ has been measured by X-ray diffraction on powder samples and is compared with the results for other zincblende-type crystals. It is shown that the data for these two compounds corroborate the linear dependence on the bond charge that has been found earlier for the effective atomic charge from infrared reflection measurements, the optical dielectric constant and the ionicity estimated from pseudopotential band theory.

Key words: Zincblende-type crystals; Bond charge; Ionicity; X-ray powder diffraction; Electron density distribution.

\section{Introduction}

The nature of the chemical bond of crystals with zincblende structure has been investigated by difference Fourier synthesis using structure factors obtained from powder samples. In the series of $\mathrm{GaP}$, $\mathrm{GaAs}$ and $\mathrm{GaSb}$, a de-like deformation becomes greater as the fifth-group atom becomes larger [1]. Part of the electrons shifted by that deformation is added to the bonding electrons so that the number of electrons in the covalent bond increases. This observation is supported by several linear relationships obtained between a measure of the number of covalent electrons and several material constants. To the above series, the zincblende-type crystals $\mathrm{ZnSe}$ and $\mathrm{ZnTe}$ are now added.

\section{Experimental}

The experimental procedures were almost the same as those used for the series of the Ga compounds [1].

\footnotetext{
* Presented at the Sagamore X Conference on Charge, Spin and Momentum Densities, Konstanz, Fed. Rep. of Germany, September 1-7, 1991

Reprint requests to Prof. Dr. Ryosei Uno, College of Humanities and Sciences, Nihon University, 3-25-40, Sakurajosui, Setagaya-ku, Tokyo 156, Japan.
}

The diffraction measurements on $\mathrm{ZnTe}$ were carried out by a newly constructed powder diffractometer with a curved-crystal $\mathrm{Si}(111)$-monochromator. In the difference Fourier synthesis anharmonic thermal vibrations were taken into account. The atomic scattering factors of the neutral atoms were used throughout. The deformation densities $\Delta \varrho(\boldsymbol{r})$ therefore represent the deviations from the electron density distribution of the superimposed spherical atoms.

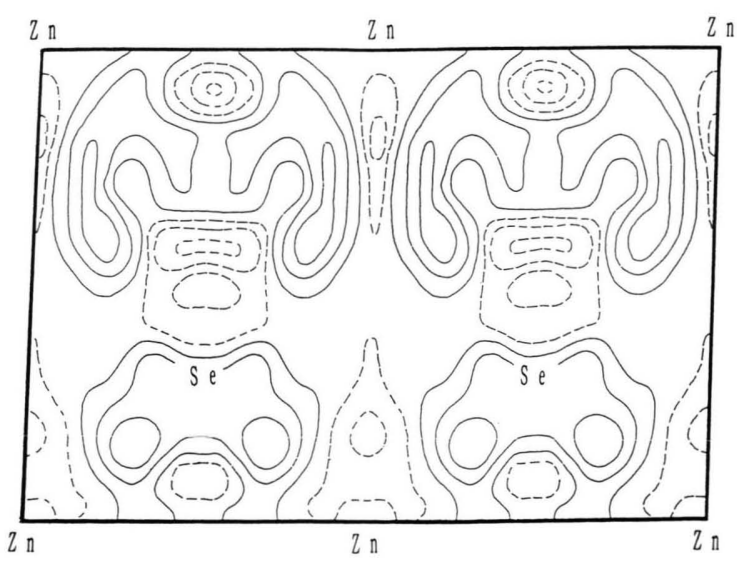

Fig. 1. The difference Fourier map $\Delta \varrho(r)$ in the (110)-plane of $\mathrm{ZnSe}$. The contour-line interval is 0.1 electrons $/ \AA^{3}$. Solid lines represent positive values, broken lines negative values. Figure 1 is distorted in process of copying. 


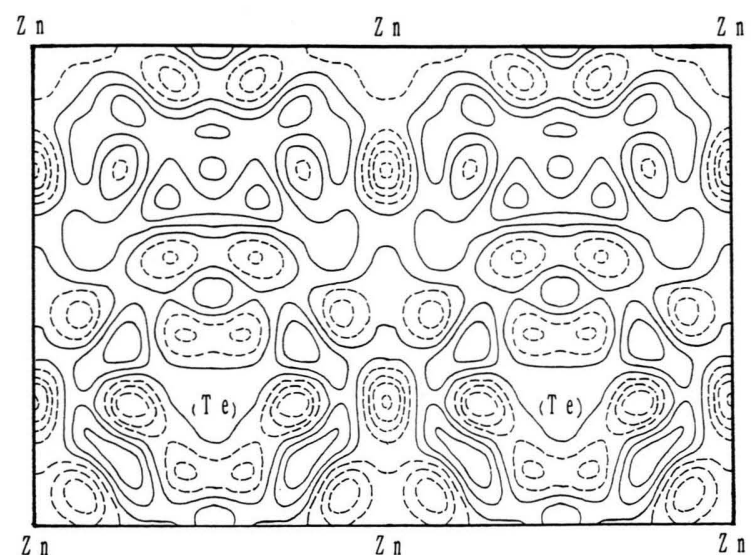

Fig. 2. The difference Fourier map $\Delta \varrho(r)$ in the (110)-plane of $\mathrm{ZnTe}$. The contour-line interval is 0.5 electrons $/ \AA^{3}$. Solid lines represent positive values, broken lines negative values.

Table 1. A measure of the number of covalent electrons, $\Delta N_{\max }$, of GaP, GaAs, GaSb, $\mathrm{ZnSe}$ and $\mathrm{ZnTe}$, and the optically obtained effective charge $e^{*}$ [2], the optical dielectric constants $\varepsilon$ [3], and the ionicity $f_{i}$ estimated by pseudopotential band theory [4].

\begin{tabular}{llllll}
\hline Crystal & GaP & GaAs & GaSb & ZnSe & ZnTe \\
\hline$\Delta N_{\max }$ & 0.06 & 0.19 & 0.47 & 0.10 & 0.23 \\
$e^{*}$ & 0.24 & 0.20 & 0.15 & 0.34 & 0.27 \\
$f_{i}$ & 0.33 & 0.31 & 0.26 & 0.63 & 0.61 \\
$\varepsilon$ & 9.1 & 10.9 & 14.4 & 5.9 & 7.3 \\
\hline
\end{tabular}

\section{Results and Discussion}

The maps of $\Delta \varrho(r)$ in the (110)-plane of $\mathrm{ZnSe}$ and $\mathrm{ZnTe}$ are shown in Figs. 1 and 2. The results for $\mathrm{ZnTe}$ are not as accurate as those for $\mathrm{ZnSe}$ because of limitations imposed by the apparatus. A peak of $\Delta \varrho(\boldsymbol{r})$ appears near the center of the chemical bond but not as clear as in the corresponding $\mathrm{Ga}$ compounds. The number of electrons, $\Delta N$, inside a sphere around the center of the chemical bond in the $\Delta \varrho$-map was calculated as a function of the radius of the sphere in the same way as described in [1]. The maximum value $\Delta N_{\max }$ of $\Delta N$ is taken as a measure of the number of electrons in the covalent bond. In Fig. 3, several mate-

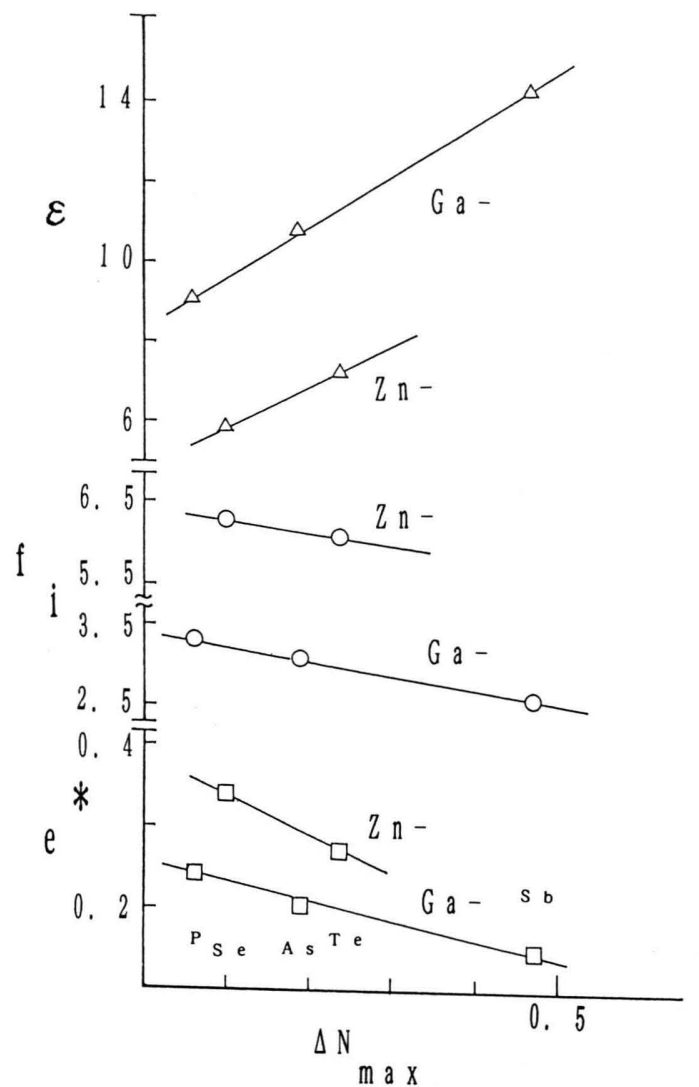

Fig. 3. Linear relationships between $\Delta N_{\max }$ and several material constants, including the optically obtained effective charge $e^{*}$ [2], the optical dielectric constant $\varepsilon$ [3], and the ionicity $f_{i}$ of the chemical bond estimated by pseudopotential band theory [4].

rial constants, such as the effective charge $e^{*}$ obtained from infrared reflection measurements [2], the optical dielectric constant $\varepsilon$ [3], and the ionicity $f_{i}$ estimated by pseudopotential band theory [4], are plotted against the $\Delta N_{\max }$ of the respective crystal. The numerical data are shown in Table 1. Each graph consists of two nearly parallel straight lines, one being for the Ga compounds and the other for the $\mathrm{Zn}$ compounds. Although the data for $\mathrm{ZnTe}$ are not so accurate, the nearly parallel two lines in Fig. 3 show that the effect of deformation of the electron cloud of the sixth-group atoms is similar to that of the fifth-group atoms. Around the center of the $\mathrm{Zn}$ atom, $\Delta \varrho(\boldsymbol{r})$ is negative in both crystals. This situation shows that some elec- 
trons are shifted away from the $\mathrm{Zn}$ atom and increase the ionicity of the bond. A Madelung-type ionic potential of the $\mathrm{Zn}$ atom may reduce the deformation of the sixth-group atom and diminish the number of electrons in the covalent bond.

[1] R. Uno, J. Ishigaki, H. Ozawa, and K. Yukino, Japan. J. Appl. Phys. 28, 573 (1989).

[2] M. Haas and B. W. Hevis, J. Phys. Chem. Solids 23, 1099 (1962).
Acknowledgement

The authors are much indebted to the many students for the help in preparing the samples and in the measurements.

[3] W. A. Harrison, Electronic Structure and the Properties of Solids, W. H. Freeman and Company, San Francisco 1980, p. 124.

[4] J. C. Phillips, Bonds and Bands in Semiconductors, Academic Press, New York 1973, p. 42. 